(Aus der anatomisch-physiologischen Abteilung des Landwirtschaftlichen Instituts der Universität Halle a. S., unter Leitung von Professor Disselhorst.)

\title{
Vergleichende Untersuchungen über den Bau und die funktionelle Anpassung der Sehnen.
}

\author{
Von \\ Otto Dammann, \\ Küniglicher Kreistierarzt in Halle a. S. \\ Mit Tafel VI und VII. \\ Eingegangen am 8. Juli 1908.
}

Das straffe, geformte fibrilläre Bindegewebe oder Sehnengewebe, wie wir es in Zukunft der Kürze halber nennen wollen, ist sowohl hinsichtlich seiner Struktur als auch bezüglich der Elemente, aus denen es sich zusammensetzt, und der Entstehung seiner Bestandteile, besonders der Fibrillen, seit langer Zeit Gegenstand eingehender und zahlreicher Untersuchungen gewesen.

An den allgemeinen Untersuchungen hieriber beteiligten sich besonders J. MüLler, der dem *Bindegewebe " seinen Namen gab, Schwann, Reichert (1) u. v. a.

Die Entstehung bzw. Bildung der Fibrillen des Bindegewebes ist besonders von Roltet, Boll (2), Lwoff (9), OBersteiner (13), Fleminisg (10 und 11), Kölliker (5) und Thürler (12) zum Gegenstand der Forschung gemacht worden, während die zelligen Elemente desselben von Henle, Waldeyer (6), Virchow (3), Merkel, BonNET (15) u. a. eingehend beschrieben wurden.

Am genauesten hat aber Ravvier (7) die verschiedenen Formen des Bindegewebes, insbesondere das Sehnengewebe, erforscht, dessen Struktar und histologische Feinheiten er in einer klassischen Darstellung charakterisierte.

Es genügt, hier anzuführen, daß das, was wir sehlechthin Sehnengewebe nennen, aus parallel verlaufenden Fibrillen- und Fibrillen- 
bündeln besteht, und daß sich zwischen den Fibrillenblindeln reihenartig angeordnete Flügelzellen (Sehnenzellen) befinden, die bei älteren Tieren weniger reichlich als bei jingeren vorhanden sind, wie wir dies ja bei allen jugendlichen Geweben beobachten. Sie können nach Böнm und DAvidoff (24) als die erhaltenen Reste derjenigen Zellen angesehen werden, von denen die Fibrillen gebildet wurden. Auf Sehnenquerschnitten sieht man größere und kleinere als Felder erscheinende Unterabteilungen: die Querschnitte der Fibrillenbindel. Die feinsten derartigen, welche keine besondere Gliederung mehr aufweisen, sind die primären Sehnenbündel; sie entsprechen einem Bindegewebsfibrillenbündel. Die einzelnen Fibrillen dieser primären Bündel werden durch eine interfibrilläre Kittsubstanz zusammengehalten, eine allgemeine Annahme, welche jedoch durch die Untersnchungen RAvviers in Frage gestellt wird. Zwischen den Bündeln selbst befindet sich interfasciculäre Grundsubstanz, in welche die flügelförmigen Sehnenzellen eingebettet sind, die ihre Gestalt ihrer Lage verdanken, nämlich der Einklemmung zwischen den verschiedenen Fibrillenbündeln. Im Querschnitt zeigen sich jene Zellen daher meist dreistrahlig sternförmig; in der Mitte haben sie einen ovalen Kern. So legen sich eine Anzahl primärer Bündel zusammen zu einem sekundïren, an dessen Oberfläche sich ein mehr oder weniger vollständiger Endotheluberzug befindet, und in dessen Licken die Grundsubstanz, welche zuweilen zahlreiche Sehnenzellen enthält, zutage tritt. Das Ganze wird umgeben von einer Hülle ungeordneten fibrillären Bindegewebes, dem Peritenonium internum. Die sekundären Bündel legen sich dann wieder zu tertiären zusammen, die von etwas dickeren Ztigen des Peritenonium umgeben werden, und durch weitere Aneinanderlagerung entsteht schließlich die Sehne, die auf ihrer Oberfläche von der stärkeren Hülle des Peritenonium externum umschlossen wird.

Bei entsprechender Behandlung der Schnitte mit Essigsäure sieht man noch ein außerordentlich feines Netz ron clastischen Fasern, welches die primären Bündel umgibt.

Blutgefäße, Lymphgefäße und Nerven verlaufen im Peritenonium internum (SCHIEFFERDECKer und Kossel, 23).

Sind somit die Struktur des Sehnengewebes an sich und alle histologischen Feinheiten desselben genau bekannt und vielfach beschrieben, so muß es anderseits wundernehmen, daß vergleichende Untersuchungen uber die Histologie desselben bisher nicht gemacht oder wenigstens nicht veröffentlicht wurden. Das ist um so auffal- 
lender, als gerade bei unsern Arbeitstieren, besonders beim Pferd und Rind, den Sehnen ein großes physiologisches und biologisches Interesse entgegengebracht werden maß. In der umfangreichen, mir zu Gebote stehenden Literatur vermochte ich nichts VergleichendHistologisches iber die Struktur der Sehne aufzufinden. Lediglich einige ganz kurze, mehr gelegentliche Andeutungen weisen darauf hin, daß gewisse Verschiedenheiten bestehen können; so sagt ToLDT (14), ohne weitere Beweise hierfür beizubringen, nach einer vorausgegangenen Beschreibung der Sehnenstruktur: »alle die genannten Verhältnisse sind natürlich an den verschiedenen Sehnen, ja an verschiedenen Stellen einer einzigen Sehne, manchen Schwankungen unterworfen". Nach RanviER (7) sowie nach einem Referate aus Merkel und Bonvet (15) stoßen die Sehnenzellen, welche Scheiden um die Sehnenbündel bilden, bei jungen, im Wachstum begriffenen Tieren unmittelbar zusammen, während sie sich bei alten Tieren voneinander entfernen. Nach MaLl (16) verhalten sich die Sehnen von verschiedenen Orten desselben Tierkörpers teilweise verschieden gegen Zusatzfliissigkeiten. Triepel (17) hält es anderseits iiberhaupt für unwahrscheinlich, daß wesentliche qualitatire ${ }^{1)}$ Unterschiede zwischen verschiedenen Sehnen, etwa Verschiedenheiten in ihrem feineren Ban, bestehen ".

Mit diesen kurzen Andeutungen ist die gesamte mir bekannt gewordene Literatur über die vergleichende Histologie des Sehnengewebes erschöpft. Es erschien daher eine dankenswerte Aufgabe, vergleichende histologische Untersuchungen über das Sehnengewebe anzustellen, und dabei auch das physikalische Verhalten der Sehnen zu berücksichtigen.

Von den mannigfaltigen Gesichtspunkten, die sich hierbei ergeben, konnten natürlich nicht alle Berücksichtigung finden; es sollen in der nachstehenden Arbeit lediglich folgende auf das Pferd und Rind beziigliche Fragen gepruft werden:

1) Hat das Sehnengewebe beim Pferd, wenngleich nach demselben Prinzip gebaut, eine andre Struktur als beim Rind? Ist insbesondere bein Pferd die Struktur eine dichtere, insofern als die Fascikel enger aneinander gelagert sind, während das interfasciculäre Bindegewebe in geringerer Menge vorhanden ist? Bestehen ferner Unterschiede in der Dicke der elementaren Fibrillen und in der Größe der Querschnittsbilder der Fascikel?

1) Das Wort > qualitativ ist hier in anderm Sinne gebraucht wie weiter unten. 
2) Bestehen Unterschiede der in 1 angegebenen Art zwischen dem Sehnengewebe edler, *warmblïtiger " Pferde und zwischen den Sehnen »kaltblitiger *?

3) Bestehen ceteris paribus solche Strukturunterschiede zwischen Sehnen von Tieren, welche gearbeitet haben, und solchen, die nicht gearbeitet haben?

4) Lassen sich solche Strukturunterschiede zwischen den Sehnen der Vorderschenkel und denen der Hinterschenkel nachweisen?

5) Kann man Strukturunterschiede zwischen den Sehnen älterer und jugendlicher, in der Entwicklung begriffener Tiere überzeugend dartun?

Zur Klärung vorstehender Fragen wurden genau korrespondierende Stellen der Hufbein- bzw. Klauenbeinbeugesehnen untersucht.

6) Hat bei ein und demselben Tier der Querschnittsquotient (das Verhältnis des Sehnenquerschnittes zum Querschnitt des zugehörigen Muskels einen Einfluß auf die Struktur der Sehnen im Sinne der Frage 1? Sind also Strukturunterschiede hinsichtlich der verschiedenen Sehnen bei demselben Tier nachweisbar?

7) Bestehen Unterschiede des spezifischen Gewichts zwischen den Sehnen, und zwar zwischen den Sehnen von edlen und denen von gemeinen kaltblutigen Pferden, zwischen den Sehnen älterer und jüngerer Tiere der gleichen Art, zwischen den Sehnen von Arbeitstieren und solchen, die nicht gearbeitet haben? Sind Unterschiede in dieser Hinsicht zwischen den Vorderschenkelund Hinterschenkelsehnen vorhanden? Hat der Querschnittsquotient einen Einfluß auf das spezifische Gewicht?

Ich bemerke noch ausdrücklich, daß sich die histologischen Untersuchungen über die Punkte $1-6$ nicht auf die feinsten Details des Sehnengewebes erstrecken sollen, sondern rorwiegend die Lagerungsverhältnisse und Dicke der Fascikel, und die Quantitat des eingelagerten interfasciculären Bindegewebes im Ange haben.

\section{Untersuchungsmethoden.}

Als Untersuchungsmaterial dienten vorwiegend Sehnen der Hufbein- bzw. Klauenbeinbeuger (M. flexor digitorum profundus s. perforans) von Kälbern, Kuihen, Ochsen, sehr edlen warmblütigen ostpreußischen Pferden, hannöverschen Halbblutpferden und von schweren kaltblütigen belgischen Pferden. 
Es wurden zum Zwecke sowohl der histologischen Untersuchung an Querschnitten, als auch zur Bestimmung des spezifischen Gewichts genau korrespondierende Stellen der in Frage kommenden Sehnen gewählt, and za dem Ende Sehnensticke von der Mitte des Metacarpus bzw. Metatarsus anfangend bis zu einem Punkte etwa $5 \mathrm{~cm}$ unterhalb dieser Stelle herausgeschnitten. Die Auswahl gerade dieser Stellen erschien aus mehrfachen Gründen zweckentsprechend; an böher gelegeneu Sehnenpartien stört beim Pferd das die Sehnen verstärkende Unterstuttzungsband, einmal deshalb, weil es, wie auch die Sehne selbst, gerade im oberen Teile häufig erkrankt, weiterhin bei Aufnahme des spezifischen Gewichts - weil sich viel lockeres Bindegewebe in der spitzwinkligen Übergangsstelle des Unterstittzungsbandes in die Sehne befindet, was die genaue Bestimmung des spezifischen Gewichts stören mußte. Näher am ersten Zehengelenk gelegene Sehuenpartien erschienen anderseits deshalb zur Untersuchung. ungeeignet, weil bei der Anspannung der Sehnen hier nicht ausschließlich eine Zugwirkung, sondern gleichzeitig eine Druckwirkung auf die Sehne stattfindet, welcher Umstand das Untersuchungsergebnis hätte beeinträchtigen können.

Außer den genannten Sehnen wurden noch Stiicke aus der Achillessehne und Strecksehnenstücke vom Vorderschenkel untersucht.

Die Fixierung erfolgte nach Rawrtz $\{20\}$ in steigender 0,3 bis $1{ }_{0}^{0}$ iger wässeriger Chromsüurelösung; danach grïndliches Auswaschen in fließendem Wasser, völliges Entwässern in steigendem,

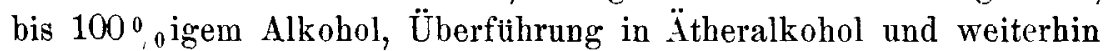
Einbettung in Celloidin. Paraffin eignet sich nicht zur Einbettung (SCHMORL, 21).

Auch Fixation nach ReTteren in Pikrinsublimat und gesättigter Pikrinsäurelösung mit Zusatz von $20_{0}^{\circ}$ Seesalz (jodhaltig), direkte Überftihrung in Alkohol ohne Entwässern, danach $̈$ theralkohol, wurden ausgeführt.

Stets wurden jedoch nur Sebnenschnitte miteinander verglichen, die nach derselben Methode fixiert waren, weil immerhin eine Möglichkeit vorlag, daß das Gewebe im ganzen, oder einzelne Bestandteile desselben unter der Einwirkung der einen Fixierungsflissigkeit mehr Einschrumpfung erleidet als unter der der andern.

Das spezifische Gewicht wurde in bekannter Weise vermittels der hydrostatischen Wage durch genaue Wägungen auf Milligramme festgestellt bzw. berechnet. Hierbei war besondere Sorgfalt erforderlich. Die zu prïfenden Sehnen mußten wegen ihrer schnellen Ein- 
trocknung sofort nach der Entnahme aus dem Tierkörper in Staniolpapier eingewickelt werden, um eine Wasserverdunstung von der Oberfläche zu vermeiden. Einwickeln in Pergamentpapier genügt nicht, da dasselbe immerhin etwas Wasser aufnimmt und so ungenaue Resultate entstehen. Wie schnell Sehnen eintrocknen, mag daraus hervorgehen, daß $10 \mathrm{~g}$ schwere Stiicke auf der Wage schon innerhalb einer Minute etwa 5 Milligramm an Gewicht abnahmen.

\section{Eigne Untersuchungen.}

Zur Frage 1. Gibt es Strukturunterschiede zwischen den entsprechenden Sehnen vom Pferd und Rind?

Ich stelle diese Frage voran, einmal mit Rucksicht darauf, daß histologische Unterschiede zwischen den Sehnen der verschiedenen Tierarten von vornherein am ehesten zu vermnten waren. Wenn sich solche Unterschiede nicht einmal hier fanden, so war vorweg anzunehmen, daß man sie innerhalb der einzelnen Tierart, bei den einzelnen Rassen erst recht nicht erwarten durfte.

An den meisten Organen der verschiedenen Haustierarten, auch an deren Muskulatur, finden sich ja Verschiedenheiten in der Gewebsstruktur vor, oft so auffalleṇder Art, daß, wie beispielsweise an der Lunge des Pferdes und Rindes oder an der Leber des Rindes und Schweines oder an der Muskulatur des Pferdes, Rindes und Schweines, sie schon makroskopisch sich erkennbar machen. Weshalb sollten solche Unterschiede nicht auch an den Sebnen auftreten?

Tatsächlich bieten denn auch die Sehnen des Pferdes und Rindes hinsichtlich der Quantität und der Anordnung des eingelagerten, formlosen, interfasciculären Bindegewebes ein recht differentes histologisches Bild.

So finden wir in der Tat beim Rind das Peritenonium, insbesondere das Peritenonium internum, weit mächtiger entwickelt als selbst beim lymphatischen kaltblütigen Pferd. Die in die Sehnen eindringenden bindegewebigen Stränge sind erheblich dicker und verzweigen sich, überaus zahlreiche Anastomosen bildend, so häufig, daß man auf dem ganzen Sehnenquerschnitt ein gleichmäßiges, außerordentlich dichtes und starkes Netzwerk vorfindet, welches in seinen Maschen nur einige wenige zusammengelagerte primäre Fibrillenbündel umfaßt, so sekundäre Bündel bildend, die sich weiter durch dickere Bindegewebszüge zu tertiären Bündeln vereinigen. Oft dringen feinere Bindegewebsziige sogar bis zwischen die primären Bündel ein. Das 
formlose Bindegewebe ist so überaus reichlich vertreten, daß es den Fibrillen gegenüber fast überwiegt (vgl. Abb. 6).

Die Sebnenquerschnitte bieten dem entgegen beim Pferde ein andres Bild. Das in die Sehne eindringende Bindegewebe ist hier viel feiner gegliedert und läßt viel dünnere Balken erkennen; sodann aber, und das ist die Hauptsache, findet beim Pferde durchaus nicht eine so weitgehende dichte, filzartige Verzweigung des Peritenonium internum statt wie beim Rinde. Es senkt sich nur eine geringe Anzahl Balken in die Sehne ein, diese verästeln und verzweigen sich untereinander, Anastomosen bildend, noch einige Male. Ein dichtes, filziges Maschenwerk wie beim Rinde bilden sie jedoch nicht (Abb. 4).

Dabei findet die Verzweigung der eindringenden Bindegewebsfascikel vorwiegend in den vorderen Partien der Sehne und zwar am Vorderrande und den Seitenrändern statt; nach dem hinteren Rande zu finden sich nur ganz spärlich dünne Bindegewebsziuge, die eine sehr große Anzahl primärer Sehnenbündel umschließen. Wir können somit der Regel nach am Sehnenquerschnitt der Hufbeinbeuger zwei Partien unterscheiden, eine mit etwas dichterem Bindegewebsgeflecht und eine meistens nach dem hinteren Sehnenrande zu gelegene Partie, in der das Bindegewebsgeflecht ganz weitmaschig und zart ist und das spezifische Sehnengewebe, die Fibrillen, fast rein zutage treten.

Dieser Befund läßt die Vermutung zu, daß den Sehnen eine gewisse innere Architektonik zukommt, ahnlich wie den Knochen.

Von diesen wissen wir, daß die Knochenbälkchen eine ganz bestimmt gerichtete mehr oder weniger dichte Lagerung haben, den Druckverhältnissen entsprechend, und daß, wenn die Druckverbältnisse sich ändern, auch die Aneinanderreihung der Knochenbälkchen eine andre wird.

Bei den Sehnen kann natiurlich da, wo sie nicht etwa uber Knochenvorspriunge oder gewinkelte Gelenke hinweggehen, wie z. B. an der binteren Fesselgelenkfläche, nur eine Zugwirkung in Frage kommen. Und es entsteht die Vermutung, daß da, wo auf dem Sehnenquerschnitt das spezifische Sehnengewebe am reinsten vertreten ist, also dort, wo sich am wenigsten formloses Bindegewebe rorfindet, die Stelle des stärksten Zuges zu suchen ist. So ergibt sich eine ungezwungene Erklärung phylogenetischer Art für diese Erscheinung. Die Zugwirkung hat in gleicher Weise schon seit vielen Jahrtausenden eingewirkt und daher an den bezeichneten Partien als die 
zweckmäBigste Struktur das Sehnengewebe am reinsten und dichtesten angehäuft.

Zur Frage 2. Wie wir gesehen haben, sind strukturelle Unterschiede des Sehnengewebes bei verschiedenen Tierarten tatsächlich vorhanden. Es entsteht die Frage, ob solche Unterschiede auch innerhalb derselben Tierart durch fortgesetzte Zuichtung nach bestimmten, möglichst divergenten Zuchtrichtungen in die Erscheinung treten. Die größten Abweichungen in dieser Hinsicht bietet wohl das englische Vollblutpferd und die schwereren kaltblutigen Schläge. Sehnen von reinem Vollblut standen mir leider erst bei Abschluß dieser Arbeit zur Verfügung; es wurden deshalb Sehnen von dem Vollblut nahestehenden sehr edlen ostpreußischen Pferden mit denen von kaltblütigen, schweren belgischen verglichen.

Unter Tierärzten und Tierzüchtern herrscht die ungeteilte Ansicht, daß die Sehnen bei edlen Pferden fester, härter und straffer seien als bei gemeinen Pferderassen. Man hört oft von den sstahlharten « Sehnen der Vollblutpferde im Vergleich zu den lockeren der kaltblutigen Pferde reden. Tatsache ist, daß die Sehnen edler Pferde - ich habe besonders die Sehnen der Zehenbenger im Auge - bei der Palpation wesentlich fester und härter erscheinen und sich ron der Umgebung schärfer absetzen, als dies bei den gleichen Sehnen der kaltblutigen Pferde der Fall ist. Es war deshalb zu prüfen, ob dies bei der Palpation verschiedene Verhalten außer durch stärkere Spannung infolge des größeren Muskeltonus tatsächlich mitbedingt ist durch eine veränderte Struktur, durch eine dichtere Aneinanderlagerung der tätigen Substanz der Sehnenfibrillen infolge Schwindens des interfasciculären Bindegewebes (Rovx, 19, I. S. 174).

Verneinendenfalls würde eine ungezwungene Erklärung hierfür auch wohl dadurch gegeben werden können, daß bei kaltblitigen Pferden erfahrungsgemäß die Hant dicker und das subcutane Gewebe stärker entwickelt ist, die Finger somit durch eine dickere Gewebsschicht hindurchpalpieren müssen, welche weicher ist als Sehnengewebe, so daß hierdurch eine geringere Härte und Festigkeit der Sehnen lediglich vorgetäuscht werden kaun.

Eine Klärung der Verbältnisse, ob die Sehnen edler Pferde tatsächlich in dem angedeuteten Sinne histologisch anders beschaffen sind, oder ob dies nur so scheint, erschien daher geboten.

Hinsichtlich der Dicke der Haut wurde bei vier zu diesem Zwecke untersuchten geschlachteten bzw. verendeten edlen ostpreußischen und 
einem westfälischen Halbblutpferd festgestellt, daß die Hant bei ihnen nur $1 / 2$ bis $2 / 3$ so dick sich erwies, als bei acht daraufhin untersuchten dänischen und belgischen Pferden. Selbst ganz kleine polnische Pferde hatten eine dickere Haut als edle ostpreuBische.

Ebenso verhielt es sich mit dem subcutanen und dem zwischen den Sehnen befindlichen lockeren Bindegewebe bei kaltblïtigen Pferden; es war in jedem Falle weit stärker und massiger ausgebildet als bei den edleren Rassen.

Es finden sich also in dieser Hinsicht nicht unwesentliche Unterschiede ror.

Vergleicht man herausgeschnittene Sehnenstiicke von edlen und kaltblütigen Pferden, so läßt sich ein auffälliger Unterschied hinsichtlich der Festigkeit auf diesem Wege nicht mehr feststellen. Dennoch aber bestehen histologische Strukturverschiedenheiten, die jedoch im Verhältnis zu den Unterschieden zwischen Pferde- und Rindersehnen geringerer Art sind.

Die Dicke der Fibrillen und der primären Bündel ist bei edlen Pferden und Kaltblittern annähernd die gleiche, auch die ganze Art der Verästelung des Peritenonium internum läBt wesentliche Unterschiede nicht erkennen. Beim näheren Vergleich einer Anzahl von Sehnenquerschnitten tritt jedoch unzweidentig zutage, daß die Bindegewebszüge, die die sekundären, tertiären und weiteren Bündel umgeben, beim Kaltblutpferde regelmäßig stärker entwickelt sind, daß das Sehnengewebe kaltblütiger Pferde somit tatsächlich mehr formloses Bindegewebe enthält, als das edler warmblütiger Rassen (Abb. 1 und 2).

Zur Frage 3. Während vorstehend die Möglichkeit der Verschiedenheit bzw. Verbesserung des Sehnengewebes infolge fortgesetzter zielbewuBter Züchtung ins Auge gefaßt wurde, ergibt sich die Berechtigung der Frage 3 aus andern Erwägungen heraus.

Es kommt hier das Prinzip der Veränderung von Organen und Geweben durch die Wirkung des Gebrauchs oder Nichtgebrauchs (LAMARCK), das Gesetz der funktionellen Anpassung (Roux) in Frage. Roux versteht darunter eine Anpassung an die Funktion durch Ausiibung der Funktion.

Schon DarwiN (26) räumt der Wirkung des Gebrauchs oder Nichtgebrauchs neben der natürlichen Zuchtwahl eine große Bedeutung ein, und erkennt damit ein Prinzip an, welches im Gegensatz zur natiirlichen Zuchtwahl das Zweckmäßige direkt hervorbringt. Daß 
die künstliche Zuchtwahl in dieser Hinsicht natürlich schnellere Erfolge auch nach andern Gesichtspunkten zeitigt, braucht nicht besonders erörtert zu werden.

Wie bei den Muskeln, so macht sich auch bei den Sehnen die funktionelle Anpassung, die Anpassung an vermehrte Arbeit (bei den Sehnen natürlich passiver Art) durch vermehrte Arbeit als Wachstum in die Dicke bemerkbar; das ist bekannt und schon grob anatomisch feststellbar. Es geschieht dies durch Neubildung von elementaren Fibrillen (Hyperplasie). Ob dabei auch eine geringgradige Verdickung der einzelnen Fibrillen (Hypertrophie) stattfindet, wie es ZIELOxKo (27) hinsichtlich der Muskelfasern nachgewiesen hat, sei dahingestellt. Ich spreche hier nur von erwachsenen, nicht mehr im Wachstum begriffenen Tieren.

Außer dieser quantitativen, die Gestalt (auch im histologischen Sinne) beeinflussenden Wirkung der funktionellen Aupassung ist aber noch hinzudeuten auf eine bislang hinsichtlich aller Organe fast unbeachtet gebliebene "qualitativ « (Rocx) ändernde Wirkung vermehrten und verminderten Gebrauchs, auf die Erhöhung bzw. Verminderung der spezifischen Leistungsfähigkeit.

Bezïglich der Muskeln ist Derartiges von W. Hexle und Krorz (25 und 18 nachgewiesen, welche fanden, daß dasselbe Volumen Muskelsubstanz des rechten Armes --- bei Korpsstudenten, die den rechten Arm durch vieles Fechten sehr stark ausgebildet hatten $20 \%$ mehr leisten könne als beim linken. Dementsprechend definiert Roux das physiologische Gesetz der funktionellen Anpassung dahin: Durch verstärkte Tätigkeit wird die spezifische Leistungsfähigkeit der Organe erhöht.

Diese Steigerung der qualitativen Leistungsfähigkeit kann nach ihm in ihren ersten Anfängen vielleicht ohne Bildung einer besseren Struktur der tätigen Substanz einhergehen, indem sie bloß auf besserer Ausnutzung der vorhandenen Struktur, auf besserer $A b f u h r$ und besserem Ersatz des verbrauchten, auf Entfernung nicht nötiger Teile (Bindegewebe, Fett) beruht. Bei den Sehnen könnte vielleicht eine noch dichtere Zusammenfassung der Bündel durch die querverbindenden Fasern, ein Schwinden des interfasciculären Bindegewebes, wie dies schon Rocx annimmt, sowie ein Ersatz durch Fibrillen in Frage kommen. Eine weitere Steigerung der qualitativen Leistungsfähigkeit müßte dagegen mit einer Verbessernng der tätigen Struktur verbunden sein, mit einer Verbesserung des atomistischen Aufbaues der elementaren Sehnenfibrillen selbst, also der von Roux auf Grund 
von Experimenten erschlossenen spezifischen funktionellen » Metastruktur * der Sehnenfasern (Nr. 19, I. S. 187). Die Notwendigkeit der Annahme einer gewissen, wenn auch in ihrer Wirkung beschränkten qualitativen und funktionellen Anpassung ist beinahe zwingend, denn ohne funktionelle Anpassung - deren Wirkung ja natürlich im gewissen Grade auch rererbt wird - würden die tierischen Organismen dauernd auf der Stufe des angeboren Vererbten bestehen bleiben; das gilt für die Bindegewebszelle so gut wie für die Ganglienzelle.

Von diesen Gesichtspunkten aus soll hier zunächst erörtert werden, ob infolge geleisteter Arbeit außer der anatomisch sichtbaren Verdickung der Sehnen durch Neubildung von Fibrillen auch histologische Veränderungen in der Sehnenstruktur eintreten.

Eine Klärung dieser Verbältnisse hat auch für die Entscheidung tierzichterischer Fragen einen gewissen Wert. Man fordert heutzutage vielfach, daß Deckhengste, welche ja in den Landesgestiiten den größten Teil des Jahres ủber ohne Beschäftigung stehen, arbeiten sollen, und begriundet das damit, daß die Sehnen und alle Gewebe durch Arbeit fester und leistungsfähiger werden, und daß sich ja diese erworbene Eigenschaft, bis zu einem gewissen Grade wenigstens, vererbt, während umgekehrt bei dauernder Untätigkeit der Tiere ein Lockererwerden und Erschlaffen der Gewebe und damit auch des Sehnengewebes mit allen seinen Konsequenzen hinsichtlich der Vererbung zu befürchten sei. Histologische Vergleiche in dieser Beziehung beim Pferd anzustellen, war mir unmöglich; Sehnen eines Pferdes, das nicht gearbeitet hat, standen mir nicht zur Verfügung. Es wurden deshalb Klauenbeinbeugesehnen von Stallkühen mit denen von Arbeitsochsen verglichen. Das Ergebnis war, daß histologische Unterschiede hinsichtlich der Quantität oder Anordnung des eingelagerten, formlosen Bindegewebes oder der Fibrillendicke nicht ermittelt werden konnten.

Man darf daraus nicht schließen, daß feine Strukturunterschiede infolge geleisteter Arbeit iberhaupt nicht eintreten; denn sie könnten sich so langsam ausbilden und daher so gering bleiben, daß sie als morphologische Veränderungen innerhalb des individuellen Lebens des betreffenden Tieres nicht mit Sicherheit histologisch nachweisbar sind.

Eine so große Bedeutung, als manche Züchter dem Punkte beilegen, daß Zuchthengste arbeiten sollen, scheint er somit hinsichtich des einzelnen verwendeten Zuchttieres nicht zu habeu. 
Die funktionelle Anpassung der Sehnen an die Arbeit macht sich in erster Linie durch Dickenwachstum der Sehne, durch Fibrillenneubildung, bemerkbar.

$\mathrm{Ob}$ daneben auch qualitative Veränderungen der Fibrillen hinsichtlich des atomistischen Aufbaues in Frage kommen können, das soll weiter unten erörtert werden.

Zur Frage 4. Die bei Frage 3 erörterten Gesichtspunkte waren auch für die vorliegende Frage maßgebend. Als Material dienten Hufbeinbeugesehnen vom Vorder- und Hinterschenkel schwerer belgischer Arbeitspferde. Abgesehen von der Rubestellung besteht ja die Wirkung der Hufbeiubenger als aktives Bewegungsorgan vorn wie hinten in einer Beagung des dritten Zehengliedes; sie beteiligen sich nach Sussnonf (28) an dem kraftvollen Eingreifen der Zehe in den Boden. Dabei ist indessen zu berlicksichtigen, daß die Sehne des Hinterschenkels, zumal bei Pferden, die schwere Lasten zu ziehen haben, Dehnungen bzw. Zugwirkungen in ungleich größerem Maße ausgesetzt ist, als die entsprechende Sehne am Vorderschenkel. Es kommen somit auch hicr etwaige Veränderungen der Sehnenstruktur durch verstärkte Inanspruchnahme in Frage.

Untersuchungsergebnis: Augenfällige histologische Unterschiede zwischen den Sehnen derVorder-und Hinterschenkel finden sich nicht. Es gilt somit auch hier das bei 3 Gesagte, daB histologische Veränderungen des Sehnengewebes durch vermehrte Arbeit während des individuellen Lebens nicht nachweisbar sind.

Zur Frage 5. Bestehen Strukturunterschiede zwischen den Selnen älterer und jugendlicher, in der Entwicklung begriffener Tiere?

Zum Vergleich dienten Klauenbeinbeugesehnen junger, etwa 4 Wochen alter Kälber mit denen 6-8jähriger Ochsen und Kühe.

Nach Obersteiner (29) schreitet die Entwicklung der Sehnen bis ungefähr um die Mitte des Embryonallebens fort; in dieser Zeit haben sie ein Ansehen erlangt, das im allgemeinen im Verlaufe der ferneren Entwicklung unverändert bleibt. RANvier (30) hat außerdem darauf hingewiesen, daß bei jugendlichen Tieren (Külbern) die Sehnenzellen näher zusammenliegen und vielfach miteinander anastomosieren, und daß der Zellleib bei ihnen dicker und protoplasmareicher ist. Bei älteren Tieren sind die Sehnenzellen weiter voneinander entfernt, der Zellleib ist mehr schuppenartig, homogen.

Ich fand diese Angaben bestätigt. Abgesehen von einer gewissen 
Untersuchungen über den Bau und die funktionelle Anpassung der Sehnen. 361

Dickenzunahme der Bündel bei fortschreitendem Wachstum der Tiere waren Strukturunterschiede zwischen Kalbssehnen und Sehnen älterer Ochsen und Kühe nicht nachweisbar.

Indessen findet man regelmäßig tinktorielle Unterschiede. Von den ïblichen Methoden, die zur Färbung von Celloidinschnitten angewandt zu werden pflegen, eignete sich keine sonderlich gut. Insbesondere gab die Hansersche Färbung (33) und die Weigertsche Hämatoxylinfärbung (31) ganz verschwommene Bilder; leidliche Resultate wurden mit der rax GIesoxschen Methode (32) erzielt. Sehr deutlich differenzierte sich dagegen bei Sehnenschnitten aller untersuchten Tiere das formlose Bindegewebe von den Fibrillen durch Färbung mit Bismarckbraun. Hierbei erscheint ersteres in Form scharf markierter dunkelbrauner Züge, während die Fibrillen hellgelb aussehen. Nur auf Sehnenschnitten von jungen Tieren (Kälbern) trat diese scharfe Markierung nicht deutlich in die Erscheinung. Das Bild erschien hier mehr verschwommen, wenngleich die Bindegewebsztige immerhin erkennbar waren. Das war auch nach der vax Gresonschen Methode der Fall.

Aus der mehr gleichmäßigen Fürbung der einzelnen Bindegewebsbestandteile bei jugendlichen Kälbern glaube ich schließen zu können, daß bei ihnen noch nicht eine so vollkommen ausgesprochene Differenzierung der einzelnen Gewebselemente, insbesondere der Sehnenfibrillen, sowie des formlosen Bindegewebes und der Sehnenzellen stattgefunden hat, als beim erwachsenen Rind, bei dem die einzelnen Bestandteile schon längere Zeit, ihrer Funktion entsprechend, tätig gewesen sind. Man kann aus dem erwähnten tinktoriellen Verhalten vielleicht auf eine qualitativ etwas andre Beschaffenheit der jugendlichen Fibrillen, welche durch Ausübung der Funktion noch nicht verändert sind, gegenüber den Fibrillen älterer Tiere schließen, vermutlich begründet durch den molekularen Aufbau der Substanz der Elementarfibrillen, also ihrer Metastruktur, oder durch abweichendes chemisches Verhalten. Diese Vermutung stimmt, wie ich gleich vorwegnehmen will, auch mit dem physikalischen Befunde überein, denn die Sehnen junger Tiere sind konstant spezifisch leichter als die älterer Tiere der gleichen Art.

6. Hat der Querschnittsquotient (das Verhältnis des Sehnenquerschnitts zum zugehörigen Muskelruerschnitt einen Einfluß auf die Struktur der Sebnen im Sinne der Frage 1? Haben somit verschiedene Sehnen desselben Tieres eine voneinander abweichende Struktur? 
Rovx sagt darüber (19, I. S. 174): „Ein nur scheinbares Beispiel qualitativer Anpassung bietet die Dicke der Sehnen dar. Ich fand, daß bei schwachem Mnskel die Sehne etwa $1 / 30$, bei den stärksten Muskeln aber (Soleus) bloß $1 / 120$ der Dicke des Muskels mißt (beide Dicken natürlich rechtwinkelig zur Faserrichtung gemessen, was bei den dicken, gefiederten Muskeln nur unter Zerlegung in viele einzelne Stiicke möglich ist). Doch beruht diese Erscheinung wohl nicht auf qualitativer Anpassung der Sehnenfasern, denn die einzelne Faser wird wohl nicht stärker gezogen, sondern bloß auf dichterer Zusammenfassung der Fasern durch die quer verbindenden Fasern der Sehne, welche bei den dicken Muskeln mehr gespannt werden [dies infolge der stärkeren Konvergenz der Muskelfasern]. Auch findet sich fast dasselbe Verbältnis wie beim Soleus an der Sehne des [dinnen] Supinator longus s. brachioradialis, da dessen Sehnenfasern durch die Armfascie zusammengedrängt werden, was auch schon beim Soleus selber geschieht. Diese geringere Dicke der Sehne als die des Muskels ist der erste Grund der Fiederung « usw. Und weiter spricht er (S. 18) davon, daß die Sehnen, entsprechend seinem "Gesetz der dimensionalen Aktivitätshypertrophie" bei verstïrkter Funktion nur dicker, nicht länger werden.

Auf der 16. Versammlung der Anatomischen Gesellschaft im Jahre 1902 in Halle a. S. referierte Triepel über das Verhältnis zwischen Muskel- und Sehnenquerschnitt. Er bezeichnet das Verhältnis der Sehnenquerschnittsfläche zum zugehörigen Muskelquerschnitt als den Querschnittsquotienten, der höher oder niedriger sein kann. TRIEPEL nimmt an, daß der Querschnitt einer Sehne in einer gewissen $A$ blängigkeit von der Aktion des zugehörigen Muskels und somit auch von seinem Querschnitte steht, und daß der Muskelquersehnitt durch andauernde Übung um das Mehrfache vergrößert werden kann, während die Sehne dabei zwar auch an Dicke, aber in relativ viel geringerem Grade zunimmt und höchstens das Doppelte des ursprünglichen Querschnitts erreicht.

TRIEPEL folgert daraus entsprechend Rouxs an verschiedenen Muskeln gewonnenem Befund für verschiedene Dicke desselben Muskels, daß bei wachsendem Muskelquerschnitt auch der Querschnittsquotient ansteigen muB.

Dieser Satz ist zweifellos richtig, sofern man nur eben je einen bestimmten Muskel bei demselben Individuum im Auge hat.

Da Triepel Unterschiede bezüglich des histologischen Baues der einzelnen Sehnen, etwa Unterschiede in der Qualität des Gewebes, 
die die Verschiedenheiten der Querschnittsquotienten ausgleichen könnten, leugnet, so folgert er, daß der Quotient Aufschluß darüber gibt, welche Stellung ein Muskel sowohl innerhalb des individuellen Lebens, als auch in phylogenetischer Hinsicht einnimmt. Diese Schliisse sind, wie schon FICK betonte, zweifellos zu weitgehend.

Einmal kommen bei der Dicke der Sehnen noch andre Nomente in Frage: Raumersparnis, Formenschönheit und das Prinzip der Bildung der zweckmäßigsten Struktur. Wo Raum für relativ dicke Sehnen, für Sehnen mit kleinem Querschnittsquotienten vorhanden ist, da sind tatsäcblich die Sehnen im allgemeinen auch relativ dicker, allerdings lockerer, und da ist auch der Quotient ein niedrigerer. Wo aber, um Raumersparnis und Formenschönheit zugleich zu erreichen, an Raum gespart werden muB, da ist die Sehne der Regel nach dünner. Zum Teil konmt hierbei die Form und Aneinanderlagerung der Skeletknochen in Frage. An der Vorderfläche des Metacarpus erscheinen nur flache, düne Sehnen zweckmäßig, dagegen bietet der Raum beispielsweise zwischen dem Fersenbeinhöcker und der Hinterfläche der Tibia Platz genug für bedeutende Entwicklung. Das Schönheitsprizzip ist ja am Tierkörper im allgemeinen auch hinsichtlich der Muskelinsertion durchgefiuhrt. Die Insertion der Muskeln ist, wie bekannt, bezüglich der Erreichung einer größtmöglichen Kraftleistung außerordentlich ungtinstig, so zwar, daß der Hebelarm der Last um ein Vielfaches länger ist als der Hebelarm der Kraft. Dadurch wird die Kraftwirkung zwar kleiner, aber die Bewegungen sind schnell und behend. Wäre es umgekehrt, so würden die Gliedmaßen unförmige, fleischige, langsam bewegliche Gebilde darstellen - eine Tatsache, die schon HrrTu überzeugend nachgewiesen hat. Die Zugkraft, der eine Sehne bei stärkster Kontraktion des zugehörigen Muskels ausgesetzt ist, entspricht im wesentlichen dem Muskelquerschnitt, da sehr erhebliche Unterschiede hinsichtlich der Qualität der Muskelsubstanz kaum in Frage kommen. Wie Henle und KNonz nachwiesen, kann sie durch ausgiebigste Übung nur etwa um ein Finftel verbessert werden. Demgegenüber sind die Unterschiede zwischen den einzelnen Querschnittsquotienten wesentlich größere, sie schwanken, wie wir sahen, beim Menschen nach Roux zwischen 1:30 und $1: 120$, also um das Vierfache. Beim Pferde fand ich Querschnittsquotienten von $1: 14$ bis $1: 47$, es fanden sich somit Schwankungen am das 3,3 fache. Der Querschnittsquotient bei den Mm. gastrocnemii und soleus betrug zum Beispiel beim Pferd 1:14, der vom M. extensor digitorum communis des Vorderschenkels $1: 47$. 
Dementsprechend hat eine gleich große Querschnittsfläche der Sehne vom M. extensor digitorum communis die $3,3(47: 14=3,3)$ fache Zugkraft auszuhalten als die von der Achillessehne.

Von obigem Gesichtspunkte aus erschien die Klärung der Frage interessant, ob das Prinzip der funktionellen Selbstgestaltung des Zweckmäßigen, der zweckmäßigen Struktur (Rovx), auch auf die Sehnen Anwendung finde, d. h. ob nicht eine Sehne mit größerem Querschnittsquotienten, die also bei gleich großen Querschnittsflächen stärker durch $\mathrm{Zng}$ in Anspruch genommen wird, ein zweckmäßigeres, besseres, weniger formloses Bindegewebe enthaltendes Gefitge bekommt als eine Sehne, die geringerer Zugkraft ausgesetzt ist. Daß innerhalb des individuellen Lebens ceteris paribus an ein und derselben Sehne solche Unterschiede histologisch nicht nachweisbar sind, habe ich bereits bei Frage 3 erörtert. Hier kommen aber auch noch phylogenetische Momente in Betracht.

Ich stellte zunächst den Querschnittsquotienten von verschiedenen Muskeln bei drei Pferden fest. Da direkte Messungen wegen der unregelmäßigen Form der Querschnittsfläche nicht wohl angängig waren, wurden die Flächenwerte nach KxoRz (18) berechnet durch Division des Volumens, welches sich aus der Wasserverdrängung ergab, mit der Länge der ausgeschnittenen Muskel- bzw. Sehnenstücke. Bei stark gefiederten Muskeln ist dies Verfahren naturlich nicht ohne weiteres anwendbar.

Ich erhielt auf diese Weise folgende Werte:

1. Belgisches Pferd:

Hufbeinbeuger vom Vorderschenkel . . . . . . $1: 19$

- Hinterschenkel . . . . . $1: 18$

Mm. gastrocnemii und soleus . . . . . . . . $1: 14$

M. extensor carpi radialis longus et brevis . . . $1: 33$

M. extensor digitorum communis vom Vorderschenkel 1:28

2. Hannöverscher Hengst:

Hufbeinbeuger rom Vorderschenkel . . . . . 1:20

- Hinterschenkel . . . . . . 1:20

Mm. gastrocnemii und soleus . . . . . . . . $1: 14$

M. extensor carpi radialis longus et brevis . . . $1: 37$

M. extensor digitorum communis vom Vorderschenkel 1:47

3. Landpferd, dän ische Kreuzung:

Hufbeinbeuger vom Vorderschenkel . . . . . . 1:21

- Hinterschenkel . . . . . . $1: 19$ 
Untersuchungen über den Bau und die funktionelle Anpassung der Sehnen. 365

Mm. gastrocnemii und soleus . . . . . . . . $1: 15$

M. extensor carpi radialis longus et brevis . . . $1: 35$

M. extensor digitorum communis vom Vorderschenkel 1:38

(Das Unterstiutzungsband der Hufbeinbeugesehnen vom Vorderschenkel wurde nicht mitgemessen, beim Hinterschenkel kommt es ja ohnehin wegen seiner Dtinne kaum in Betracht.)

Die gewonnenen Zahlen können naturlich auf mathematische Genauigkeit keinen Anspruch machen; das liegt schon in der Methode der Querschnittsmessungen. Aber es ist doch leicht zu ersehen, daß von den in Frage stehenden Muskeln der Quotient bei den Mm. gastrocnemii und soleus am kleinsten, bei den Strecksehnen am höchsten ist, und daß die Hufbeinbeuger in der Mitte zwischen beiden stehen.

Analog verbält es sich mit der Quantität und Verteilung des den betreffenden Sehnen eingelagerten Bindegewebes. Die untersuchten Sehnen vom M. extensor digitorum communis des Pferdes haben nur Spuren von formlosem Bindegewebe in sich, das auf dem Sehnenquerschnitt etwa wie ein weitmaschiges, ganz feines Spinngewebe aussieht; bei den Hufbeinbeugesehnen findet man schon ein deutliches Gerïstwerk mit dickeren und dünneren, wenn auch immerhin lockeren Verzweigungen; die Achillessehne aber ist durchzogen von einem recht dicken, filzigen Bindegewebsgeflecht, und kommt hierin schon der Klauenbeinbeugesehne des Rindes nahe (Abbild. 3, 4 und 5 ).

Es hat danach den Anschein, als ob das Prinzip der funktionellen Selbstgestaltung der zweckmäBigen Struktur gerade in den Sehnen recht deutlich in die Erscheinung tritt. Sehnen mit kleinen Querschnittsquotienten, die also einen relativ geringeren $Z u g$ auszuhalten haben, sind mit viel formlosem Bindegewebe durchsetzt, während bei den Sehnen, die den stärksten Zugwirkungen ausgesetzt sind, das spezifische Sebnengewebe fast in voller Reinheit zutage tritt.

\section{Unterschiede des spezifischen Gewichts.}

Wenn man beruicksichtigt, daß die Sehnen aus verschiedenen elementaren Bestandteilen, der Hauptmasse nach aus sehr festgefügten Fibrillenbündeln spezifischen Sehnengewebes und aus eingelagertem lockerem Bindegewebe zusammengesetzt sind, so muß, wenn die Voraussetzung zutrifft, daß das eigentliche Sehnengewebe als »tätige «, 
festgefügte Substanz spezifisch schwerer ist als lockeres Bindegewebe, gefolgert werden, daß durch ein verschiedenes Mischungsverbältnis dieser Bestandteile auch Veränderungen des spezifischen Gewichts herbeigeftuhrt werden können.

Von dieser Erwägung ansgehend wurde das spezifische Gewicht von Sehnen einer größeren Anzahl Tiere gepriff. Vergleiche über die erhaltenen Werte haben hierbei naturgemäß nur innerhalb derselben Tierart eine gewisse Bedentung. Ich stellte vermittels der hydrostatischen Wage folgende spezifischen Gewichte fest:

1) Kalb, neugeboren:

Klauenbeinbeugesehne vom Vorderschenkel 1103, - Hinterschenkel 1105.

2) Kalb, 10 Tage alt:

Klauenbeinbeugesehne vom Vorderschenkel 1104,

3) Kalb, 14 Tage alt:

- Hinterschenkel 1108.

Klauenbeinbengesehne vom Vorderschenkel 1102,

- Hinterschenkel 1102.

4) Kalb, 4 Wochen alt:

Klauenbeinbeugesehne rom Vorderschenkel 1104,

- Hinterschenkel 1109.

5) Kalb, 8 Wochen alt:

Klauenbeinbeugesehne vom Vorderschenkel 1106,

- Hinterschenkel 1108.

6) Junger Stier, 2 Jahre alt:

Klanenbeinbeugesehne vom Vorderschenkel 1117,

- Hinterschenkel 1135.

7) Kuh, 5 Jahre, Holländer:

Klanenbeinbeugesehne vom Vorderschenkel 1128, - Hinterschenkel 1147.

8) Kah, 6 Jahre, Holländer:

Klauenbeinbeugesehne vom Vorderschenkel 1139,

- Hinterschenkel 1146.

9) Bayrischer Arbeitsochse, 6 Jahre:

Klauenbeinbeugesehne vom Vorderschenkel 1117,

- Hinterschenkel 1144.

10) Bayrischer Arbeitsochse, 8 Jahre:

Klauenbeinbeugesehne vom Vorderschenkel 1127,

- Hinterschenkel 1147. 
Untersuchnngen über den Ban und die funktionelle Anpassung der Sehnen. 367

11) Belgisches Pferd, 12 Jahre:

Hufbeinbeugesehne vom Vorderschenkel 1110,

12) Belgisches Pferd, 15 Jahre:

Hufbeinbeugesehne vom Vorderschenkel 1114,

- Hinterschenkel 1122.

13) Belgisches Pferd, 15 Jahre:

Hufbeinbeugesehne vom Vorderschenkel 1116,

- Hinterschenkel 1124.

14) Hannöversches Pferd, 14-16 Jabre:

Hufbeinbengesehne vom Vorderschenkel 1118,

- Hinterschenkel 1122.

15) Hannöversehes Pferd, etwa 18 Jahre:

Hufbeinbeugesehne vom Vorderschenkel 1116,

$$
\text { - } \quad \text { - Hinterschenkel } 1124 .
$$

16) Litauer Pferd, 15 Jahre:

Hufbeinbeugesehne rom Vorderschenkel 1115,

- Hinterschenkel 1119.

17) Ostpreußisches Pferd, 8 Jahre, sehr edel gezogen:

Hufbeinbengesehne rom Vordersehenkel 1118,

- Hinterschenkel 1138.

18) Englisches Vollblutpferd Helm XX, 15 Jahre alt (Mutter von Hammurabi).

Hufbeinbeugesehne vom Vorderschenkel 1122,

- Hinterschenkel 1142.

ferner:

19) Belgier, 8 Jahre:

Huf beinbeugesehne vom Vorderschenkel. . . . . 1115

(Quotient 1:19)

Hufbeinbengesehne vom Hinterschenkel . . . . . 1119

(Quotient 1:18\}

Achillessehne . . . . . . . . . . . . . . 1119

(Quotient 1:14)

Sehne vom M. extensor carpi radialis longus et brevis 1129

(Quotient 1:33)

Sehne vom M. extens. digit. comm. des Vorderschenkels 1138

(Quotient 1:28).

20) Hannöverscher Hengst, 15 Jahre:

Hufbeinbeugesehne vom Vorderschenkel. . . . . 1114

(Quotient 1:20) 
Hufbeinbeugesehne vom Hinterschenkel . . . . 1120

(Quotient 1:20)

Achillessebne .

(Quotient 1:14)

Sehne vom M. extensor carpi radialis longus et brevis 1133

(Quotient 1:37)

Sehne vom M. extens. digit. comm. des Vorderschenkels 1130

(Quotient 1:47).

Vergleicht man die gefundenen Zahlen, so ergibt sich zunächst, daß das spezifische Gewicht mit zunehmendem Alter ansteigt. Während es z. B. an Klauenbeinbeugesehnen bei Kälbern 1102-1109 beträgt, haben die gleichen Sehnen bei jungen Stieren schon ein spezifisches Gewicht von 1117-1135, und ältere Kiuhe und Ochsen zeigen sogar ein solches von 1117-1147. Auffallend ist auch die Tatsache, daß die genannten Beugesehnen des Hinterschenkels konstant spezifisch etwas schwerer sind als die des Vorderschenkels. Bei Pferden ist hervorzuheben, daß das spezifische Gewicht gleicher Sehnen bei edlen Pferden wesentlich höher ist als beim Kaltbluter; allgemein sind auch hier die Hufbeinbeugesehnen der Hinterschenkel spezifisch schwerer als die der Vorderschenkel.

Bezüglich des Querschnittsquotienten ist eine absolute Regelmäßigkeit insofern nicht festzustellen, als das spezifische Gewicht nicht genau mit dem Querschnittsquotienten steigt. Im allgemeinen finden sich aber auch hier die höheren Werte an denjenigen Sehnen, die im Verhältnis zum Muskel dünn, also großen Zugwirkungen ansgesetzt sind.

\section{Schlußfolgerungen.}

Fassen wir die gewonnenen Ergebnisse insgesamt kurz zusammen, so ergibt sich hinsichtlich des ron mir untersuchten Materials im wesentlichen folgendes:

Die Sehnen des Rindes enthalten sehr viel mehr lockeres Bindegewebe als diejenigen des Pferdes.

In den Sehnen edler, warmblütiger Pferde ist weniger formloses Bindegewebe enthalten als in denen kalthlitiger, doch sind die Unterschiede nicht so groß als zwischen Pferd und Rind im allgemeinen.

Die Verteilung des lockeren Bindegewebes ist der Regel nach auf einem und demselben Sehnenquerschnitt nicht überall eine gleich- 
mäßige; gewöhnlich findet man eine Partie, in der das formlose Bindegewebe nur spärlich auftritt gegeniiber andern Partien desselben Querschnitts. Es muß angenommen werden, daß in diesen die Stelle des stärksten Zuges liegt, daß somit der Sehne eine gewisse innere, aus der Funktion abzuleitende Architektonik zukommt.

In den Sehnen sehr junger, in der Entwicklung begriffener Tiere (Kälber) hat noch nicht eine so vollkommen ausgesprochene, ihrer Funktion entsprecheude Differenzierung der einzelnen Gewebsbestandteile stattgefunden, als in den Sehnen älterer erwachsener Tiere.

Unterschiede zwischen Sehnen arbeitender und nicht zur Arbeit verwendeter Tiere lassen sich ceteris paribus innerhalb des individuellen Lebens histologisch nicht nachweisen.

Sehnen mit relativ schwachem zugehörigen Muskel enthalten viel lockeres Bindegewebe; bei Sehnen mit relativ starkem Muskel tritt dagegen das spezifische Sehnengewebe fast in voller Reinheit zutage. Das spezifische Gewicht der Sehnen älterer Tiere derselben Art ist höher, als das in der Entwicklung begriffener und jugendlicher Tiere; auch zeigen sich innerhalb derselben Tierart Unterschiede bezüglich züchterisch sehr weit auseinanderstehender Rassen. So sind z. B. die Sehnen ron Vollblut- und edlen warmbliutigen Pferden spezifisch schwerer als die ron kaltbliutigen.

Sehnen mit zugehörigem relativ kräftigen Muskel haben im allgemeinen ein höheres spezifisches Gewicht, als solche mit schwachem zugehörigen Muskel.

Die aufgefundenen gestaltlichen Verschiedenheiten im Baue der Sehnen fallen also zum größeren Teile in Rouxs erste causale Periode der Ontogenese des betreffenden Organs, in die Periode der rein durch die Vererbung bedingten selbständigen Gestaltungen, nur zum kleineren Teile dagegen in die Periode der durch die Ausübung der Funktion bewirkten Gestaltungen (19a, I. S. 180; 19b, S. 94).

Die vorstehende Arbeit wurde aut Veranlassung und unter Leitung des Herrn Universitätsprofessor Dr. Disselforonst in der anatomisch-physiologischen Abteilung des Landwirtschaftlichen Instituts der Universität Halle a. S. ausgeführt. Es ist mir eine angenehme Pflicht, auch an dieser Stelle Herrn Professor Dr. Disselhonst für die Unterstützung und das Interesse, das er meinen Arbeiten entgegenbrachte, sowie für die zahlreichen Anregungen bei Ausfuhrung der Arbeiten verbindlichst zu danken. 
Auch dem Leiter der hiesigen Waisenhaus-Apotheke, Herrn Dr. Fromme, welcher mir bei Feststellung der spezifischen Gewichte behilflich war, spreche ich meinen aufrichtigen Dank aus.

\section{Literaturverzeichnis.}

1) Reichert, Bemerkungen zur vergleichenden Naturforsehung und vergleichende Beobachtungen ïber das Bindegewebe und die verwandten Gebilde. Dorpat 1845 .

2) BoLL, Untersuchungen ïber den Ban und die Entwicklung der Gewebe. Archiv f. mikr. Anatom. 1871. 1872.

3) Virchow, Verhandl. d. phys.-med. Gesellsch. zn Würzburg. 1851.

4) Munck. Physiologie des Menschen und der Haustiere.

5) Kölliker, Handbuch der Gewebelehre.

6) WaLdeyer, Über Bindegewebszellen. Archiv f. mikr. Anatom. 1874.

7) Ravvier. Les éléments et les tissus du systẻme conjonctive. Leçons, faites au Collège de France. Journ. de Micrographie. 1889. 1890. 1891. 1892.

8) Ellenberger und Günther, Grundriß der vergleichenden Histologie der Haussäugetiere.

9) Lwoff, Über die Entwicklung der Fibrillen des Bindegewebes. Sitzungsber. d. K. K. Akad. Wien. Math.-nat. Kl. Bd. 98. Abt. III.

10) Flemmixg, Zur Entwicklungsgeschichte der Bindegewebsfibrillen. Internationale Beiträge zur wissenschaftl. Medizin. Bd. I. Berlin 1891.

11) — Kern und Zellteilung. Leipzig 1882.

12) ThürLer, Stadien ïber die Funktion des fibrösen Gewebes. Inauguraldissertation. Zürich 1884.

13) Obersteiner, Über Entwicklung und Wachstum der Sehnen. Sitzungsber. d. math.-naturwiss. Klasse d. Kais. Akad. d. Wissenschaften. Jahrg. 1867. Bd. 56. Abt. II.

14) ToLdT, Lehrbuch der Gewebelehre. Stuttgart 1884.

15) Merkel und Bonnet, Ergebnisse der Anatomie und Entwicklungsgesehichte. Bd. I. Wiesbaden 1892.

16) Math, Das reticulierte Gewebe und seine Beziehungen zu den Bindegewebsfibrillen. Abhandl. d. Kgl. Sächs. Gesellsch. d. Wissensch. Bd. 17. Nr. IV. Leipzig 1891.

17) H. TRIEPEL. Über das Verhältnis zwischen Muskel- und Sehnenquersehnitt. Verhandl. d. Anatom. Gesellsch. auf d. 16. Versamml. in Halle a. S. vom 22.-25. April 1902. Abgedrackt im Anatom. Anzeiger. Jena 1902.

18. F. Kropz, Ein Beitrag zur Bestimmung der absoluten Muskelkraft. Dissertation. Marburg 1865̃. Zeitschr. f. rationelle Medizin v. Henle n. O. Pfeurer. Bd. XXIV. Leipzig u. Heidelberg 1865.

19) Roux, Gesammelte Abhandlungen iiber die Entwicklungsmechanik der Organismen. Leipzig 1895.

19a) _- Der Kampf der Teile im Organismus. 1881.

19b) — Die Entwicklnngsmechanik, ein neuer Zweig der biologischen Wissenschaft. Vortr. u. Aufs. iuber Entw.-Mech. Nr. 1. 1905.

20) Rawitz, Lehrbuch der mikroskopischen Technik. Leipzig 1907. 


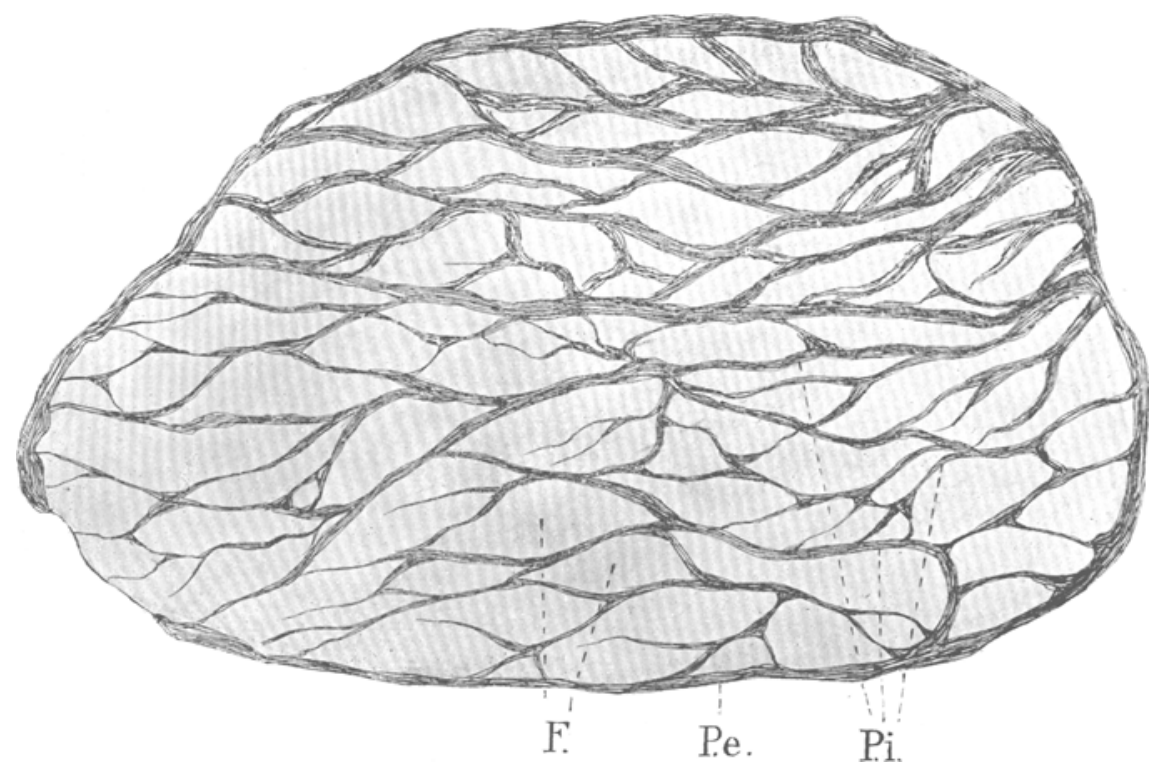

Fig. 1.

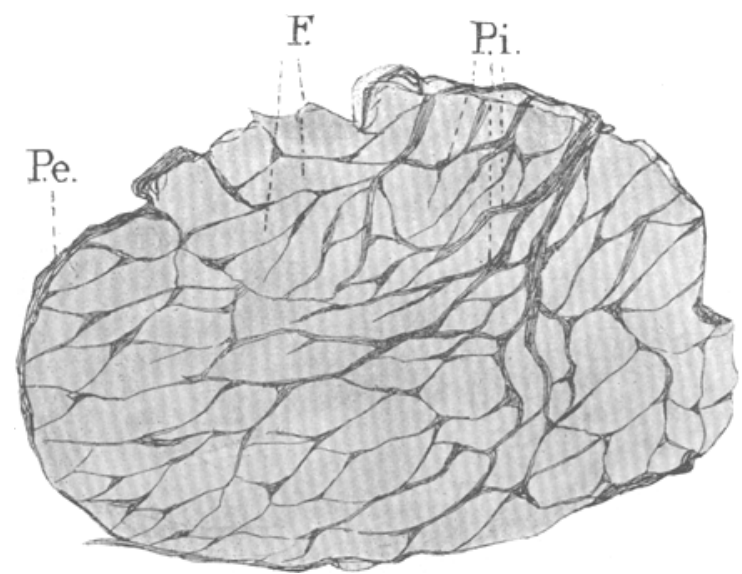

Fig. 2.

Terlig ron Wilhelm Engelmann in Leipzig. 
Untersuchungen über den Bau und die funktionelle Anpassung der Sehnen. 371

21) Schmorl, Die pathologisch-histologischen Untersuchungsmethoden. Leipzig 1905.

22) A. B. Lee und Paul Merer, Grundziige der mikroskopischen Technik. Berlin 1907.

23) Schiefwerdecker und Kossen, Gewebelehre. Braunschweig 1891.

24) Böhy und DavidofF, Lehrbuch der Histologie des Menschen. Wiesbaden 1895.

25) Hente, Zeitschrift für rationelle Medizin. Bd. XXXIII. 1868.

26) Darwix, Über das Variieren der Pflanzen und Tiere.

27) Zielonko, in Virchows Archiv. Bd. 61.

28) Sussdonf, Lehrbuch der vergleichenden Anatomie der Haustiere. Stuttgart 1895.

29) Obersteiner, Über Entwicklung und Wachstum der Sehne. Sitzungsber. d. math.-naturwiss. Klasse d. Kais. Akad. d. Wissensch. Wien 1867.

30) Ranvier, Technisches Lehrbuch der Histologie. Übersetzt von Wrss und NiCATI. Leipzig 1888.

31) WeIGeRT, Über Schnittserien von Celloidinpräparaten des Centralnervensystems zum Zwecke der Markscheidenfärbung. Zeitschr. f. wiss. Mikroskopie. Bd. 2.

32) van Gieson, zitiert nach v. Kahloen: Technik der histologischen Untersuchung pathologisch-anatomischer Präparate. 4. Aufl. Jena 1895.

33) Hansen, Eine zuverlïssige Bindegewebsfärbung. Anatom. Anzeiger. Bd. 15. Jena 1899.

34) VARALdi, Sulla presenza di elementi cartilaginei spessore di tendini negli animali domestici. Parma.

\section{Erklärung der Abbildungen.}

Tafel VI und VII.

Fig. 1. Querschnitt der Hufbeinbengesehne eines belgischen Pferdes, schwach vergrößert anf Papier projiziert, die Lmrisse der Bindegewebszüge eingezeichnet. $F$. Fascikel, P.i. Peritenonium internum, P.e. Peritenonium externum.

Fig. 2. Querschnitt der Hufbeinbeugesehne eines edlen ostpreußischen Pferdes, in gleicher Weise gezeichnet. $F$. Fascikel, P.i. Peritenonium internum, P.e. Peritenonium externum.

Fig. 3. Sehne des M. extensor digitorum communis eines belgischen Pferdes Querschnitt). s. Sehnenzellen, P.i. Peritenonium internum.

Fig. 4. Hufbeinbeugesehne vom Vorderschenkel eines belgischen Pferdes QQuerschnitt). $s$ Sehnenzellen, P.i. Peritenonium internum.

Fig. 5. Achillessehne eines belgischen Pferdes (Querschnitt). s. Sehnenzellen, P.i. Peritenonium internum.

Fig. 6. Klauenbeinbeugesehne einer Kuh (Querschnitt). P.i.1. dickere, P.i.2. mittelstarke, P.i.3. diinnere, P.i.4. feinste Bindegewebsziuge des Peritenonium internum. $s$. Sehnenzellen. 